\title{
Proceso de Atención de Enfermería aplicado a una persona con trauma craneoencefálico
}

\author{
Eder A Villegas Sánchez,* Sara Santiago García **
}

\begin{abstract}
RESUMEN
Se aplicó el Proceso de Atención de Enfermería (PAE) en el hospital "Adolfo López Mateos" acorde al enfoque metodológico enfermero, empleando la teoría del déficit del autocuidado de Dorothea E. Orem, dirigido a un paciente masculino de 24 años con diagnóstico médico de politraumatismo, traumatismo craneoencefálico grado III y hemorragia subaracnoidea, con escala de valoración neurovascular Fisher II. El objetivo de este trabajo es que el profesional de enfermería adquiera opiniones, opciones, conocimientos y habilidades para diagnosticar, brindar tratamientos de enfermería e identificar los factores de riesgo que ayuden al paciente a su pronta recuperación o a mantener su estado de salud-enfermedad no sólo en el aspecto biológico, sino también en el aspecto psicosocial y espiritual, con el fin de prevenir patologías secundarias. Los aspectos teóricos están en proceso de desarrollo y perfeccionamiento. Por dichas razones, la investigación es el puente que une la teoría con la práctica y en donde la búsqueda del conocimiento es la base para formar el juicio y fundamento de las acciones de enfermería.
\end{abstract}

Palabras clave: Proceso de atención enfermero, teoría de Dorothea Orem, enfermería.

\section{Nursing Care Process applied to a person with traumatic brain injury}

\begin{abstract}
We applied the Nursing Care Process (SAP) at the Hospital "Lic. Adolfo López Mateos" nurse according to the methodological approach, using the self-care deficit theory of Dorothea E. Orem, aimed at a male patient of 24 years with physician diagnosed multiple trauma, head injury grade III subarachnoid hemorrhage, with rating scale neuro-vascular Fisher II. The aim of this work is that the nurse acquires opinions, options, knowledge and skills to diagnose, provide nursing treatments and identify risk factors that helps the patient to a speedy recovery or maintain their health and disease not only the biological aspect, but also the psychosocial aspect and spiritual in order to prevent secondary conditions. The theoretical aspects are under development and refinement. For these reasons, the research is the bridge between theory and practice where the pursuit of knowledge is the base to form the judgment and nursing actions.
\end{abstract}

Key words: Nursing process, Dorothea Orem Theory, nursing.

* Pasante de la Licenciatura en Enfermería, UNAM-FES Zaragoza.

** Mtra. EE, Profesora de Asignatura de la carrera en Enfermería, UNAM-FES Zaragoza.

Correspondencia: santiago90_03@yahoo.com.mx

Insurgentes sur 3877, Col. la Fama

Este artículo puede ser consultado en versión completa en http://www.medigraphic.com/enfermerianeurologica 


\section{INTRODUCCIÓN}

$\mathrm{E}$ 1 Proceso de Atención de Enfermería (PAE) es un método que ayuda a los profesionales a emplear sus conocimientos, solucionar problemas, desarrollar su creatividad y tener presente el aspecto humano de su profesión. Tiene como objetivo proporcionar cuidados de enfermería individualizados y permitir la interacción con el sujeto de cuidado en forma holística, es decir, en sus dimensiones biopsicosocio-cultural y espiritual. ${ }^{1}$

Con la aparición del proceso de enfermería, en los años sesenta, las enfermeras comenzaron a tener un lenguaje común que facilitó compartir aspectos de la práctica, así como evidenciar sus experiencias al dar cuidado; el enfoque procedimental cambió hacia el de una práctica basada en el conocimiento científico. ${ }^{1}$

El modelo de Dorothea Elizabeth Orem, respecto al desarrollo de la teoría enfermera del déficit del autocuidado, se basa en tres conceptos: autocuidado, déficit de autocuidado y los sistemas de enfermería. ${ }^{2}$

\section{OBJETIVO GENERAL}

Elaborar un plan de cuidados mientras el paciente esté en el Servicio de UCI, acorde a las respuestas humanas y requisitos que vaya presentando, con la utilización de las cinco etapas del Proceso de Atención de Enfermería.

\section{METODOLOGÍA}

Estudios de caso, en los que se representan situaciones o problemáticas diversas de la vida real para el análisis de datos concretos que nos permitan reflexionar, analizar y discutir en grupo las posibles salidas que se pueden encontrar a cierto problema. ${ }^{2}$

\section{DESCRIPCIÓN DEL CASO}

Se trata de paciente masculino de 24 años, de nombre Pedro (ficticio); días de estancia: 15 y aún permanece en la UCI; ocupación: estudiante de ingeniería aeronáutica; patrón de vida: hijo, estado civil soltero, religión católica, alergias negativo, tipo de sangre $\mathrm{O}$ positivo, toxicomanías positivo; con diagnóstico médico de politraumatismo, traumatismo craneoencefálico grado III, hemorragia subaracnoidea, con escala de valoración Fisher II.

Cráneo: Normoencéfalo con herida en zona parietal derecha afrontada sin datos de sangrado activo. Ojos: Pupilas anisocóricas der. $2 \mathrm{~mm}$ izq. $3 \mathrm{~mm}$ arreactivas, reflejo corneal ausente, con lesiones de escalpe facial cicatrizado. Nariz: Fractura en tabique nasal. Oídos:
Orejas pequeñas con presencia de canal auditivo y membrana timpánica sin lesiones. Boca: Edematizada con múltiples lesiones de escalpe en zona peribucal cicatrizadas, mucosas orales deshidratadas; permeable, con heridas en ambas porciones laterales de la lengua y sangrado abundante, sin ausencia aparente de dentadura. Cuello: Faringe normal cilíndrica con tráquea central sin adenomegalias, no se palpa tiroides, pulsos presentes e inmovilidad de movimientos del cuello. Tórax y pulmón: Se encuentra intubado con presión inspiratoria de 12, Peep 6, $\mathrm{FiO}^{2} 100 \%$, VC. 455 mL, VM 8.1. Campos pulmonares hipoventilados, con crepitación en hemitórax derecho, contusión pulmonar bilateral y miocárdica. Cardiovascular: Ruidos cardiacos asincrónicos con bradicardia y taquicardia, llenado capilar mayor a tres segundos, y pulsos presentes. Abdomen: Semigloboso a expensas de tejido adiposo, blando, depresible, con presencia de ruidos peristálticos disminuidos, edema generalizado. Génito: Órganos sexuales sin alteraciones y sin salida de secreciones, con sonda vesical a derivación.

Extremidades superiores e inferiores: Simétricas, tono y fuerza muscular disminuidas, con lesiones de heridas por politraumatismo y tratamiento médico, fractura del tercio medio de clavícula derecha, miembros pélvicos sin presencia de insuficiencia venosa, pliegues simianos, con edema de +++. Columna vertebral: Anormal, con fracturas: C3 a T3, clavícula derecha (tercio medio), Apófisis espinosa C7 y transversa C6, contusión medular de C3 a C6. Piel: hidratada, turgente, ligeramente ictérica, con lesiones de escalpe así como de punciones en dorso de ambas manos, tercio medio de antebrazo al igual que a nivel de arteria subclavia derecha e izquierda.

En la segunda etapa del proceso se desprenden los diagnósticos de enfermería reales, conforme los requisitos alterados y los factores de riesgo que el paciente presenta, así como la realización de intervenciones de enfermería dirigidas a la pronta recuperación o a mantener su estado de salud-enfermedad en su estancia. Con base en las problemáticas encontradas y los factores de riesgo detectados, se implementaron cuidados con el fin de mantener el estado de salud-enfermedad para corregir y mejorar su estado. ${ }^{8}$

En la ejecución se realizaron acciones de enfermería para la mejoría de la paciente, como son: administración de medicamentos, cuidados a sonda nasogástrica (SNG), sonda Foley, cuidados para prevenir úlceras por presión, monitorización hemodinámica, aspiración de secreciones por tubo endotraqueal-boca, control de líquidos y glucemias, entre otros. En la evaluación se analizaron los datos sobre cómo el paciente respondió al tratamiento y los cuidados que se le brindaron. ${ }^{9}$ 


\section{Planes estandarizados}

Indicios: FR: 28x', SPO $89 \%$, ventilador, abundantes secreciones en boca y cánula, endotraqueal fétidas, acidosis metabólica, contusión pulmonar bilateral y miocárdica, trauma cerrado de tórax, 4 hemotórax derecho con pleurostomía, campos pulmonares con sibilancias, SNG, cánula de Guedel, presión inspiratoria de 12, Peep 6, $\mathrm{FiO}_{2} 100 \%$, VC. $455 \mathrm{~mL}, \mathrm{VM}$ 8.1, PH7.01, $\mathrm{PaO}_{2}$ 27.4, $\mathrm{PaCO}_{2} 73.4, \mathrm{HCO}_{3}$ 18,2, lactato 6.4, postparo $\mathrm{PH}_{7.16}, \mathrm{PaO}_{2} 47.6, \mathrm{PaCO}_{2}$ 57.8, $\mathrm{HCO}_{3}$ 20.5, lactato 7 , Ramsay de 6.

\section{Código: 00030}

Etiqueta diagnóstica: deterioro del intercambio de gases r/c ventilación perfusión cambios en la membrana alvéolo-capilar $\mathrm{m} / \mathrm{p}$ gasometría arterial anormal, $\mathrm{pH}$ arterial anormal, respiración anormal asistencia de (ventilación mecánica)
Dominio:

Eliminación e intercambio
Clase:

Función respiratoria

Resultados esperados NOC: 0415 estado respiratorio

0402 estado respiratorio: intercambio gaseoso

0410 estado respiratorio: permeabilidad de las vías respiratorias

0403 estado respiratorio: ventilación

Indicadores

*1 Deterioro grave, 2 deterioro sustancial, 3 deterioro moderado, 4 desviación leve y 5 sin desviación.

Intervenciones NIC

Código Intervenciones

$3160 \quad$ Aspiración de las vías aéreas

- Determinar la necesidad de la aspiración oral y/o traqueal

- Auscultar los sonidos antes y después de la aspiración

- Disponer precauciones universales: guantes, gafas, y máscara, si es el caso. Hiperoxigenar con oxígeno al $100 \%$ mediante la utilización del ventilador o bolsa de resucitación manual

- Seleccionar un catéter de aspiración que sea la mitad del diámetro interior del tubo endotraqueal, tubo de traqueotomía o vía aérea del paciente

- Dejar al paciente conectado al ventilador durante la aspiración, si se utiliza un sistema de aspiración traqueal cerrado

- Observar el estado de oxígeno del paciente (niveles de $\mathrm{SaO}_{2}$ y SvO y estado hemodinámico (nivel de PAM y ritmo cardiaco), inmediatamente antes, durante y después de la succión

- Aspirar la orofaringe después de terminar la succión traqueal. Anotar el tipo y la cantidad de secreciones obtenidas

Manejo de las vías aéreas artificiales

- Disponer una vía aérea orofaríngea o una cánula de Guedel para impedir morder el tubo endotraqueal, si procede

- Proporcionar una humidificación de $100 \%$ al gas/aire inspirado

- Proporcionar una hidratación sistémica adecuada mediante la administración oral o intravenosa de líquidos

- Mantener el inflado del globo del tubo endotraqueal/cánula o traqueotomía mediante una técnica mínimamente oclusiva o una técnica de fugas mínima

- Cambiar las cintas/sujeción del globo endotraqueal cada 24 horas, inspeccionar la piel y la mucosa bucal, mover el tubo ET al otro lado de la boca

- Aflojar los soportes comerciales del tubo endotraqueal al menos una vez al día y proporcionar cuidados a la piel

- Auscultar si hay sonidos pulmonares bilaterales. Al igual que de crepitaciones y roncus

- Marcar la referencia en centímetros en el tubo endotraqueal para comprobar posibles desplazamientos

- Observar si hay descenso del volumen exhalado y aumento de la presión inspiratoria en los pacientes que reciben ventilación mecánica

- Iniciar la aspiración endotraqueal si está indicado

- Instituir medidas que impidan la desintubación accidental: fijar las vías aéreas con cinta/cuerda; administrar sedación y relajantes musculares, si procede, y disponer sujeción en los brazos, si procede

- Proporcionar cuidados bucales y aspirar orofaringe, si procede. Realizar fisioterapia torácica, si procede

3180 Manejo de la ventilación mecánica: invasiva

- Consultar con otros cuidados para la selección de ventilación (modo inicial habitualmente de control de volumen con frecuencia respiratoria, niveles de $\mathrm{FiO}_{2}$ y volumen corriente diana especificado)
Aumentar a:

Sistema de enfermería: TC

Fundamentación científica

Las vías aéreas son las responsables de la mayor parte de las resistencias a flujo aéreo. Las lesiones obstructivas a nivel de las vías altas repercutirán de forma ostensible en la ventilación pulmonar. La aspiración endotraqueal es una técnica que permite remover secreciones acumuladas en la vía aérea que no pueden ser eliminadas efectivamente por otros medios.

Se procede a aspirar a través del tubo endotraqueal y por vía orofaríngea con el fin de remover secreciones contenidas.

Antes, durante y después del proceso de aspiración es recomendable colocarle oxígeno al paciente al $100 \%$ ya que el proceso de extubación produce gran ansiedad en el paciente elevando su consumo de $\mathrm{O}_{2}$, por lo que se corre el riesgo de hipoxemia y efectos cardiovasculares indeseables.

El circuito cerrado puede incrementar la resistencia y el volumen enviado por el ventilador; también es posible que la succión sea interpretada como un esfuerzo inspiratorio del paciente y si el ventilador se encuentra en el modo asistido ciclará a la fase inspiratoria.

La presencia del catéter en la faringe seguramente producirá náuseas; si se produjera el vómito debe colocarse al paciente en decúbito lateral y aspirar rápidamente la orofarínge con el fin de evitar broncoaspiración.

Una vez ubicado el catéter en la laringe es posible que se produzcan tos, broncoespasmo o laríngoespasmo como parte del mecanismo de defensa contra un cuerpo extraño, también pueden producirse sangramientos a nivel de la mucosa nasal o faríngea.

Durante la aspiración endotraqueal las arritmias cardiacas ocurren normalmente por hipoxemia; sin embargo, también la estimulación mecánica de la vía aérea puede causarlas; adicionalmente, la estimulación vagal puede causar bradicardia y asístole; las taquicardias se asocian más a hipoxemia y agitación del paciente. 


\section{Continúa de... Planes estandarizados}

- Controlar las actividades que aumentan el consumo de $\mathrm{O}_{2}$ (fiebre, escalofríos, convulsiones, dolor o actividades básicas de enfermería) que puedan sustituir los ajustes del soporte del ventilador y causar una desaturación de $\mathrm{O}_{2}$

- Proporcionar cuidados para aliviar el distrés del paciente (posición, limpieza traqueobronqueal, terapia broncodilatadora, sedación y/o analgesia)

3300 Cuidados del drenaje torácico

- Asegurarse de que todas las conexiones de los tubos estén firmemente fijadas

- Observar periódicamente la corriente salida del tubo torácico y las fugas de aire

- Monitorizar la permeabilidad del tubo torácico

- Observar y registrar el volumen, tono, color y la consistencia del drenaje del pulmón

- Observar si hay signos de infección

- Limpiar la zona alrededor del sitio de inserción del tubo

- Asegurarse de que el pleurovac del tubo torácico se mantiene en una posición vertical.
La ventilación mecánica es un procedimiento de respiración artificial que sustituye o ayuda temporalmente a la función ventilatoria de los músculos inspiratorios. No es una terapia, es una intervención de apoyo, una prótesis externa y temporal que ventila al paciente mientras se corrige el problema.

La programación de los diferentes parámetros de ventilación mecánica tiene la función, junto a la de oxigenar y de ventilar, la de proteger a los pacientes de la posible lesión asociada, que supone la propia ventilación en el parénquima pulmonar, y favorecer la recuperación o reparación del órgano disfuncionante por la que se indicó: cerebro, corazón o pulmón.

Resultados esperados NOC: 0406 perfusión tisular cerebral

0416 perfusión tisular celular

Intervenciones NIC

Código
Intervenciones
AUMENTA A:

Sistema de enfermería: TC Fundamentación científica
2540 Manejo del edema cerebral

- Seguir de cerca el estado neurológico y compararlo con valores guía

- Monitorizar los signos vitales

- Monitorizar niveles de $\mathrm{PaO}_{2} \mathrm{PCO}_{2} \mathrm{pH}$ y bicarbonato

- Suministrar sedación. Observar los cambios del paciente en las respuestas a los estímulos

- Colocar la cama con el cabecero levantado a $30^{\circ}$ o más

- Evitar el uso del PEEP

- Administrar agentes bloqueadores neuromusculares, según proceda

- Restringir la administración de líquidos

- Evitar la administración de líquidos hipotónicos iv

- Evitar maniobras de valsalva

Mejora de la perfusión cerebral

- Consultar con el médico para determinar los parámetros hemodinámicos y mantener dichos parámetros dentro del margen debido

- Inducir la hipertensión con la expansión del volumen o con agentes inotrópicos o vasoconstrictores, según prescripción, para mantener los parámetros hemodinámicos y mantener/mejorar la presión de perfusión cerebral (PPC).

- Administrar expansores de volumen para mantener los parámetros hemodinámicos, según prescripción

- Monitorizar los tiempos de protrombina (PT) y tromboplastina parcial (PPT)

- Mantener el nivel de hematocrito alrededor de 33\% para la terapia de hemodilución hipervolémica

- Mantener el nivel de glucosa en suero dentro del margen normal.

- Administrar y vigilar los efectos de los diuréticos de asa y corticosteroides

- Administrar bloqueadores de canal de calcio según prescripción

- Observar si hay signos de hemorragia (búsqueda de sangre en heces y drenajes nasogástricos)

- Controlar la PAM, monitorizar la PVC
El edema vasogénico es el más frecuente; se produce, entre otras lesiones en traumatismos, inflamaciones, alrededor de tumores y de hemorragias y se observa principalmente en la sustancia blanca.

En parte se trata de un edema común y en parte, de un edema inflamatorio con aumento de permeabilidad vascular por alteración endotelial. El líquido se acumula principalmente en el espacio intersticial y, en menor grado, en los astrocitos.

El edema cerebral puede conducir a herniaciones de la masa encefálica y además, a detención del flujo sanguíneo con muerte cerebral. Las herniaciones pueden ser causa de muerte.

Una posición adecuada disminuirá el riesgo del incremento de la PIC.

El furosemida es un diurético y la metilprednisolona un desinflamatorio, uno disminuye el edema y el otro no deja que se acumulen líquidos que favorezcan al edema.

Deben utilizarse soluciones isotónicas, como cristaloide se empleará SSF y como coloide puede emplearse sero-albúmina al $5 \%$. No se aconsejan soluciones glucosadas excepto si hay hipoglucemia.

\begin{tabular}{lll}
\hline Fecha & Resultados obtenidos & Evaluación de todo el plan de cuidado
\end{tabular}

Ejecución $\quad$ Evaluación




\section{MARCO TEÓRICO}

\section{Teoría de enfermería del déficit de autocuidado de Orem}

Dorothea E. Orem es una de las teóricas de enfermería más destacada de Norteamérica y siempre trabajó para mejorar la formación en enfermería. ${ }^{3}$ El objetivo de la enfermería, para ella, era ayudar al individuo a mantener por sí mismo acciones de autocuidado para conservar la vida y recuperarse de la enfermedad, logrando afrontar las consecuencias de la misma. ${ }^{4}$

\section{Epidemiología del traumatismo craneoencefálico}

En México, el trauma craneoencefálico (TCE) en el año 2005 fue la cuarta causa de muerte después de las enfermedades crónico-degenerativas, enfermedades cardiacas y el cáncer. La OMS prevé que para el 2020, esta patología será uno de los diez principales problemas de salud en los países desarrollados. ${ }^{5,6,7}$

\section{DISCUSIÓN}

Con la utilización del Proceso de Atención de Enfermería podemos reconocer que el seguimiento fue útil para estructurar el pensamiento crítico enfermero, así como los aspectos físico, biológico, psicológico, emocional y espiritual del paciente. Durante el seguimiento del caso clínico, el paciente se fue deteriorando de forma gradual por su misma condición patológica, agregándosele diversas complicaciones; se brindó la opción de enseñarle a los familiares los cuidados que deberían tener cuando se diera de alta, fracasando días después tras su deceso.

De tal forma, se puede determinar que el plan de cuidados fue benéfico en ciertos momentos de la evolución del paciente, mejorando el estado de salud-enfermedad; en otras ocasiones había retroceso; sin embargo, las complicaciones fueron mermando el estado de salud y así mismo el avance del tratamiento tanto médico como enfermero.

\section{REFERENCIAS BIBLIOGRÁFICAS}

1. Noguera Ortiz, NY. Proceso de atención de enfermería: una herramienta para la garantía del cuidado. Revista de enfermería 2008 septiembre (acceso 26 de septiembre 2010); 11 (4):31-35. Disponible en: http://www. encolombia.com/medicina/enfermeria/Enfermvol110408/Procesosdeatenciondeenfermeria.htm

2. El estudio de casos como técnica didáctica. En línea: http://www.ulavirtual.cl/ulavirtual/SITIO\%20WEB\%20CRA/recursos_ensenanza/estrategiasytecnicasdidacticas/estudio_de_casos_como_tecnica_didactica. pdf, consultado el día 15 de noviembre del 2010.

3. Rojas GA, Sandra PP, Lluch BA, Maritza CA. Gestión del cuidado enfermero en la teoría del déficit de autocuidado. Revista Cubana de Enfermería, versión impresa ISSN 0864-0319. Ciudad de la Habana 2009: 25 (3-4).

4. Cabrera E. Ética del modelo de Dorotea Orem, Instituto Superior de Ciencias Médicas de La Habana (ISCM-H) Facultad de Ciencias Médicas. En línea en: http://Victor/subir/rhab/rhcm_vol_6num_3/rhcm13307.htm (1 of 11)10/07/2007 10:59:13, consultado el día 15 de noviembre del 2010.

5. En línea: http://www.sen.es/pdf/2010/guttmann_2010.pdf, consultado el día 20 de noviembre del 2010

6. Uscanga CD, Castillo LJA, Arroyo G. Hallazgos por tomografía computada en pacientes con trauma craneoencefálico, su relación con la evolución clínica y cálculo del edema cerebral. Revista Neurología, Neurocirugía y Psiquiatría 2005; 38 (1): 11-19.

7. Martínez GJ. Traumatismo craneoencefálico. Arch Invest Pediátr Méx 2005; 8 (1): 19-26.

8. Velásquez JC, Romero JD, Archila PE. Medicina interna en urgencias. Edit. Médica Celsus, Colombia 2005.

9. Fortuna CJA, Rivera MJR, Roldan GAM. Protocolo de atención del paciente grave "Normas, procedimientos y guías del diagnóstico y tratamiento". Edit. Médica Panamericana, México 2008.

10. Carrillo ER, García GM, Martínez HL. Disfunción ventricular izquierda secundaria a hemorragia subaracnoidea. Revista de la Asociación Mexicana de Medicina Crítica y Terapia Intensiva 2010; XXIV (4): 196-200.

11. Huerta MLF, Wilkins GA, Silva RR. Hemorragia subaracnoidea en el Servicio de Admisión Continua del Centro Médico Nacional Siglo XXI Than G. Med Int Mex 2010; 26 (3): 237-242.

12. Guzmán F. Fisiopatología del trauma craneoencefálico. Colombia Médica 2008; 39 (Suppl 3). 